\title{
Size and area of square lattice polygons
}

\author{
Iwan Jensen* \\ Department of Mathematics \& Statistics, \\ The University of Melbourne, Victoria 3010, Australia
}

October 29, 2018

\begin{abstract}
We use the finite lattice method to calculate the radius of gyration, the first and second area-weighted moments of self-avoiding polygons on the square lattice. The series have been calculated for polygons up to perimeter 82. Analysis of the series yields high accuracy estimates confirming theoretical predictions for the value of the size exponent, $\nu=3 / 4$, and certain universal amplitude combinations. Furthermore, a detailed analysis of the asymptotic form of the series coefficients provide the firmest evidence to date for the existence of a correction-to-scaling exponent, $\Delta=3 / 2$.
\end{abstract}

\section{Introduction}

A self-avoiding polygon (SAP) can be defined as a walk on a lattice which returns to the origin and has no other self-intersections. The history and significance of this problem is nicely discussed in [1]. Generally SAPs are considered distinct up to translations, so if there are $p_{n}$ SAPs of perimeter length $n$ there are $2 n p_{n}$ walks (the factor of two arising since the walk can go in two directions). In addition to enumerations by perimeter, one can also enumerate polygons by the enclosed area (or number of unit cells), or both perimeter and area. Of particular interest are the first few area-weighted moments of the perimeter generating function. Also of great interest is the mean-square radius of gyration, which measure the typical size of a SAP.

This paper builds on the work of Enting [2] who used transfer matrix techniques to enumerate square lattice polygons by perimeter to 38 steps. This enumeration was later extended by Enting and Guttmann to 46 steps [3] and then to 56 steps [4]. This latter work also included calculations of moments of the caliper size distribution. Hiley and Sykes [5] obtained the number of square lattice polygons by both area and perimeter up to perimeter 18. Enting and Guttmann extended the calculation to perimeter 42 [6]. The radius of gyration was calculated for SAPs up to 28 steps by Privman and Rudnick [7], using a technique based on direct counting of compact site animals on the dual lattice. Recently, Jensen and Guttmann devised an improved algorithm for the enumeration of SAPs and extended the calculation to 90 steps [8]. The work reported here is based on generalisations of this improved algorithm. This has enabled us to extend the calculation

*e-mail: I.Jensen@ms.unimelb.edu.au 
of the radius of gyration and the first two area-weighted moments to 82 step SAPs. The generalisation of the transfer matrix technique to area-weighted moments is similar to the one used by Conway [9] in his calculation of series for percolation problems and lattice animals. The generalisation to the radius of gyration has to our knowledge no counterpart in the published literature, and represents a major advance in the design of efficient counting algorithms. Previous calculations of the radius of gyration were based on direct counting algorithms. The transfer matrix algorithm used in this paper is exponentially faster and thus enables us to significantly extend the series (see [8] for further details).

The size exponent, $\nu$, for self-avoiding polygons is believed to be identical to that of self-avoiding walks. This has been argued theoretically from the connection between the energy-energy and spin-spin correlation functions of the $n$-vector model in the limit $n \rightarrow 0$, and SAPs and SAWs, respectively [10, 11]. Alternatively it has also been obtained from real space renormalization group arguments [12]. The exponent describing the growth of the mean area of polygons of perimeter $n$ is expected to be $2 \nu$ [13]. Intuitively this is not surprising since it just means that the average area of a polygon is proportional to the square of the radius of gyration. So one is merely finding that for this problem the typical area and typical length scale match one another nicely. These expectations have been confirmed reasonably accurately by numerical work [4, 6, 7].

The functions we consider in this paper are: (i) the polygon generating function, $\mathcal{P}(u)=\sum p_{n} u^{n}$; (ii) $k^{\text {th }}$ area-weighted moments of polygons of perimeter $n,\left\langle a^{k}\right\rangle_{n}$; and (iii) the mean-square radius of gyration of polygons of perimeter $n,\left\langle R^{2}\right\rangle_{n}$. These quantities are expected to behave as

$$
\begin{aligned}
p_{n} & =B \mu^{n} n^{\alpha-3}[1+o(1)], \\
\left\langle a^{k}\right\rangle_{n} & =E^{(k)} n^{2 k \nu}[1+o(1)], \\
\left\langle R^{2}\right\rangle_{n} & =D n^{2 \nu}[1+o(1)],
\end{aligned}
$$

where $\mu=u_{c}^{-1}$ is the reciprocal of the critical point of the generating function, and $\alpha=1 / 2$ and $\nu=3 / 4$ are known exactly [14, though non-rigorously. It is also known [15] that the amplitude combination $E^{(1)} / D$ is universal, and that

$$
B D=\frac{5}{32 \pi^{2}} \sigma a_{0}
$$

where $a_{0}$ is the area per site and $\sigma$ is an integer such that $p_{n}$ is non-zero only if $n$ is divisible by $\sigma$. For the square lattice $a_{0}=1$ and $\sigma=2$. These predictions have been confirmed numerically [15, 16].

In the next section we describe the generalisation of the finite lattice method required in order to calculate the radius of gyration and area-weighted moments of self-avoiding polygons. The results of the analysis of the series are presented in Section 3.

\section{Enumeration of self-avoiding polygons}

The method used to enumerate self-avoiding polygons in this work is based on the method devised by Enting [2] for enumerations by perimeter and uses the enhancements of Jensen and Guttmann [8]. In the following we first very briefly outline the original method and 
then show how to generalize it in order to calculate area-weighted moments and the radius of gyration. Details of the algorithm can be found in the papers cited above.

The first terms in the series for the perimeter generating function can be calculated using transfer matrix techniques to count the number of polygons spanning (in both directions) rectangles $W+1$ edges wide and $L+1$ edges long. The transfer matrix technique involves drawing a boundary through the rectangle intersecting a set of $W+2$ edges. For each configuration of occupied or empty edges along the boundary we maintain a (perimeter) generating function for partially completed polygons. Polygons in a given rectangle are enumerated by moving the boundary so as to add one site at a time. Due to the obvious symmetry of the lattice one need only consider rectangles with $L \geq W$. Any polygon spanning such a rectangle has a perimeter of length at least $2(W+L)$. By adding the contributions from all rectangles of width $W \leq W_{\max }$ (where the choice of $W_{\max }$ depends on available computational resources) and length $W \leq L \leq 2 W_{\max }-W+1$, with contributions from rectangles with $L>W$ counted twice, the number of polygons per vertex of an infinite lattice is obtained correctly up to perimeter $n_{\max }=4 W_{\max }+2$. The number of configurations required as $W_{\max }$ is increased grows exponentially as $\lambda^{W_{\max }}$, where $\lambda \simeq 2$ for the improved algorithm [8]. In addition to the dominant exponential growth in memory requirements there is a prefactor, which is proportional to the number of terms $n_{\max }$.

\subsection{Area-weighted moments}

Area-weighted moments can easily be calculated from the perimeter and area generating function

$$
\mathcal{C}(u, v)=\sum_{n, m} c_{n, m} u^{n} v^{m}
$$

where $c_{n, m}$ is the number of polygons with perimeter $n$ and area $m$. From this we get the area-weighted generating functions,

$$
\mathcal{P}^{(k)}(u)=\left.\left(v \frac{\partial}{\partial v}\right)^{k} \mathcal{C}(u, v)\right|_{v=1}=\sum_{n} \sum_{m} m^{k} c_{n, m} u^{n}=\sum_{n} p_{n}^{(k)} u^{n},
$$

and we define the average moments of area for a polygon with perimeter $n$

$$
\left\langle a^{k}\right\rangle_{n}=p_{n}^{(k)} / p_{n}^{(0)}=\sum_{m} m^{k} c_{n, m} / p_{n}
$$

In order to calculate the moments of area through this approach we need to calculate a full two-parameter generating function, which generally will require a lot of computer memory. If we are only interested in the first few moments there is a much more efficient approach [9]. We simply replace the variable $v$ by $1+z$ thus obtaining the function

$$
F(u, z)=\sum_{n, m} c_{n, m} u^{n}(1+z)^{m}=\sum_{n, m} \sum_{k=0}^{m}\left(\begin{array}{c}
m \\
k
\end{array}\right) c_{n, m} u^{n} z^{m} .
$$

Let, $F_{i}(u)$, be the coefficient of $z^{i}$ in $F(u, z)$. Then we see that

$$
F_{0}(u)=\sum_{n, m} c_{n, m} u^{n}=\mathcal{P}(u)
$$




$$
\begin{aligned}
& F_{1}(u)=\sum_{n, m} m c_{n, m} u^{n}=\mathcal{P}^{(1)}(u), \\
& F_{2}(u)=\sum_{n, m} m(m-1) / 2 c_{n, m} u^{n}=\left[\mathcal{P}^{(2)}(u)-\mathcal{P}^{(1)}(u)\right] / 2,
\end{aligned}
$$

and so on. Thus if we are only interested in the first and second moments of area we can truncate the series $F(u, z)$ at second order in $z$ and find the relevant moments as $\mathcal{P}^{(1)}(u)=F_{1}(u)$ and $\mathcal{P}^{(2)}(u)=2 F_{2}(u)+F_{1}(u)$. The growth in memory requirements is still dominated by the exponential growth in the number of configurations. However, we have managed to turn the calculation of these moments from a problem with a prefactor cubic in $W_{\max }$ (the area is proportional to $W_{\max }^{2}$ ) into a problem with a prefactor linear in $W_{\max }$.

\subsection{Radius of gyration}

In the following we show how the definition of the radius of gyration can be expressed in a form suitable for a transfer matrix calculation. Note that we define the radius of gyration according to the vertices of the SAP and that the number of vertices equals the perimeter length. The radius of gyration of $n$ points at positions $\mathbf{r}_{i}$ is

$$
n^{2} R_{n}^{2}=\sum_{i>j}\left(\mathbf{r}_{i}-\mathbf{r}_{j}\right)^{2}=(n-1) \sum_{i}\left(x_{i}^{2}+y_{i}^{2}\right)-2 \sum_{i>j}\left(x_{i} x_{j}+y_{i} y_{j}\right) .
$$

This last expression is suitable for a transfer matrix calculation. As usual [7] we actually calculate the generating function, $\mathcal{R}_{g}^{2}(u)=\sum_{n} p_{n}\left\langle R^{2}\right\rangle_{n} n^{2} u^{n}$. In order to do this we have to maintain five partial generating functions for each possible boundary configuration $\sigma$, namely

- $P(u)$, the number of (partially completed) polygons according to perimeter.

- $R^{2}(u)$, the sum over polygons of the squared components of the distance vectors.

- $X(u)$, the sum of the $x$-component of the distance vectors.

- $Y(u)$, the sum of the $y$-component of the distance vectors.

- $X Y(u)$, the sum of the 'cross' product of the components of the distance vectors, e.g., $\sum_{i>j}\left(x_{i} x_{j}+y_{i} y_{j}\right)$.

As the boundary line is moved to a new position each boundary configuration $\sigma$ might be generated from several configurations $\sigma^{\prime}$ in the previous boundary position. The partial generation functions are updated as follows

$$
\begin{aligned}
P(u, \sigma) & =\sum_{\sigma^{\prime}} u^{n\left(\sigma^{\prime}\right)} P\left(u, \sigma^{\prime}\right) \\
R^{2}(u, \sigma) & =\sum_{\sigma^{\prime}} u^{n\left(\sigma^{\prime}\right)}\left[R^{2}\left(u, \sigma^{\prime}\right)+\delta\left(x^{2}+y^{2}\right) P\left(u, \sigma^{\prime}\right)\right] \\
X(u, \sigma) & =\sum_{\sigma^{\prime}} u^{n\left(\sigma^{\prime}\right)}\left[X(u, \sigma)+\delta x P\left(u, \sigma^{\prime}\right)\right] \\
Y(u, \sigma) & =\sum_{\sigma^{\prime}} u^{n\left(\sigma^{\prime}\right)}\left[Y(u, \sigma)+\delta y P\left(u, \sigma^{\prime}\right)\right] \\
X Y(u, \sigma) & =\sum_{\sigma^{\prime}} u^{n\left(\sigma^{\prime}\right)}\left[X Y\left(u, \sigma^{\prime}\right)+\delta x X\left(u, \sigma^{\prime}\right)+\delta y Y\left(u, \sigma^{\prime}\right)\right]
\end{aligned}
$$


where $n\left(\sigma^{\prime}\right)$ is the number of occupied edges added to the polygon and $\delta=\min \left(n\left(\sigma^{\prime}\right), 1\right)$.

\subsection{Further particulars}

Finally a few remarks of a more technical nature. The number of contributing configurations becomes very sparse in the total set of possible states along the boundary line and as is standard in such cases one uses a hash-addressing scheme. Since the integer coefficients occurring in the series expansions become very large, the calculation was performed using modular arithmetic. Up to 8 primes were needed to represent the coefficients correctly. Further details and references are given in [8]. The series for the radius of gyration and area-moments were calculated for SAPs with perimeter length up to 82 . The maximum memory required for any given width did not exceed $2 \mathrm{~Gb}$. The calculations were performed on an 8 node Alpha Server 8400 with a total of $8 \mathrm{~Gb}$ memory. The total CPU time required was about three days per prime. Obviously the calculation for each width and prime are totally independent and several calculations were done simultaneously.

In Table 1 we have listed the series for the radius of gyration and first and second area-weighted moments. The series for the radius of gyration of course agree with the terms up to length 28 computed previously [7], while the terms up to length 40 for the first area moment agree with the series in [6]. The number of polygons of length $\leq 56$ can be found in [0] while those up to length 90 were reported in [8].

\section{Analysis of the series}

The series listed in Table 1 have coefficients which grow exponentially, with sub-dominant term given by a critical exponent. The generic behaviour is $G(u)=\sum_{n} g_{n} u^{n} \sim(1-$ $\left.u / u_{c}\right)^{-\xi}$, and hence the coefficients of the generating function $g_{n} \sim \mu^{n} n^{\xi-1}$, where $\mu=$ $1 / u_{c}$. To obtain the singularity structure of the generating functions we used the numerical method of differential approximants [17]. In particular, we used this method to estimate the critical exponents (we already have very accurate estimates for $u_{c}$ from [8]). Since all odd terms in the series are zero and the first non-zero term is $g_{4}$ we actually analysed the function $F(u)=\sum_{n} g_{2 n+4} u^{n}$. Combining the relationship given above between the coefficients in a series and the critical behaviour of the corresponding generating function with the expected behaviour (1) of the mean-square radius of gyration and moments of area yields the following prediction for their generating functions:

$$
\begin{aligned}
\mathcal{R}_{g}^{2}(u) & =\sum_{n} p_{2 n+4}\left\langle R^{2}\right\rangle_{2 n+4} n^{2} u^{n}=\sum_{n} r_{n} u^{n} \sim R(u)\left(1-u \mu^{2}\right)^{-(\alpha+2 \nu)}, \\
\mathcal{P}^{(k)}(u) & =\sum_{n} p_{2 n+4}\left\langle a^{k}\right\rangle_{2 n+4} u^{n}=\sum_{n} a_{n}^{(k)} u^{n} \sim a^{(k)}(u)\left(1-u \mu^{2}\right)^{2-(\alpha+2 k \nu)} .
\end{aligned}
$$

Thus we expect these series to have a critical point, $u_{c}=1 / \mu^{2}=0.14368062927(1)$, known to a very high degree of accuracy from the analysis in [8], and as stated previously the exponent $\alpha=1 / 2$, while it is expected that $\nu=3 / 4$.

Estimates of the critical point and critical exponents were obtained by averaging values obtained from second order $[L / N ; M ; K]$ inhomogeneous differential approximants. For each order $L$ of the inhomogeneous polynomial we averaged over those approximants to the series which used at least the first $80 \%$ - $90 \%$ of the terms of the series. We 
used only approximants where the difference between $N, M$, and $K$ didn't exceed 2 . Some approximants were excluded from the averages because the estimates were obviously spurious. The error quoted for these estimates reflects the spread (basically one standard deviation) among the approximants. Note that these error bounds should not be viewed as a measure of the true error as they cannot include possible systematic sources of error. In Table 2 we have listed the results of our analysis. It is evident that the estimates for $u_{c}$ and the critical exponents are in agreement with the expected behaviour. There are only some minor discrepancies in the fourth digit between the conjectured exponents and the estimates. This discrepancy is readily resolved by looking at the evidence in figure 1, where we have plotted the estimates for the critical point and exponent of $\mathcal{R}_{g}^{2}$. Each point in these figures represent an estimate obtained from a specific second order differential approximant with the various points obtained by varying the order of the polynomials in the approximants. It is clear that the estimates have not yet settled down to their asymptotic values and that they do converge towards the expected values as the number of terms used by the approximants is increased.

Now that the exact values of the exponents has been confirmed we turn our attention to the "fine structure" of the asymptotic form of the coefficients. In particular we are interested in obtaining accurate estimates for the amplitudes $B, D$ and $E^{(1)}$. We do this by fitting the coefficients to the assumed form (11).

The asymptotic form of the coefficients $p_{n}$ of the polygon generating function has been studied in detail previously [18, 8]. As argued in [18] there is no sign of non-analytic corrections-to-scaling exponents to the polygon generating function and one therefore finds that

$$
p_{n}=\mu^{n} n^{-5 / 2} \sum_{k=0} a_{k} / n^{k}
$$

This form was confirmed with great accuracy in [8]. Estimates for the leading amplitude $B=a_{0}$ can thus be obtained by fitting $p_{n}$ to the form given in equation (11). In order to check the behaviour of such estimates we did the fitting using from 2 to 10 terms in the expansion. The results for the leading amplitude are displayed in figure 2. We notice that all fits appear to converge to the same value as $n \rightarrow \infty$, and that, as more and more correction terms are added to the fits the estimates exhibits less curvature and that the slope become smaller (although the fits using 10 terms are a little inconclusive). This is very strong evidence that (11) indeed is the correct asymptotic form of $p_{n}$. We estimate that $B=0.5623012(1)$.

The asymptotic form of the coefficients $r_{n}$ in the generating function for the radius of gyration has not been studied previously. When fitting to a form similar to equation (11), assuming that here are only analytic corrections-to-scaling, we find that the amplitudes of higher order terms are very large and that the leading amplitude converge rather slowly. This indicates that this asymptotic form is incorrect. We find that the coefficients fit better if we assume a leading non-analytic correction-to-scaling exponent $\Delta=3 / 2$. This result confirms the prediction of Nienhuis [14. Note, that since the polygon generating function exponent $2-\alpha=3 / 2$ a correction-to-scaling exponent $\Delta=3 / 2$ is perfectly consistent with the asymptotic form (11). Because $2-\alpha+\Delta$ is an integer the nonanalytic correction term becomes part of the analytic background term [18]. We thus propose the following asymptotic form: 


$$
r_{n}=\mu^{n} n\left[B D+\sum_{k=0} a_{k} / n^{k / 2}\right]
$$

Alternative we could fit to the form

$$
r_{n} / p_{n}=n^{7 / 2}\left[D+n^{5 / 2} \sum_{k=0} a_{k} / n^{k / 2}\right]
$$

In figure 3 we show the leading amplitudes resulting from such fits while using from 1 to 10 terms in these expansions. Also shown in these figures (solid lines) are the predicted exact value of $B D$, given in equation 2, and the prediction for $D$ using the estimate for $B$ obtained above. As can be seen the leading amplitudes clearly converge towards their expected values and from these plots we can conclude that the prediction for $B D$ has been confirmed to at least 6 digit accuracy. Assuming that equation (2) is exact and using the very accurate estimate for $B$ we find that $D=0.05630944(1)$.

Fitting the coefficients for the area-weighted moments to asymptotic forms similar to equations (12) and (13) above (only the leading exponent was changed accordingly) leads to the estimates $E^{(1)}=0.141520(1)$ and $E^{(2)}=0.0212505(4)$.

As stated above the analysis of the polygon generating function is fully consistent with the prediction $\Delta=3 / 2$. However, all one can conclude from the analysis is that, if non-analytic correction-to-scaling terms are present, the exponents have to be "halfinteger", so that the correction terms become part of the analytic background. The detailed analysis of the asymptotic form of the coefficients in the generating functions for the radius of gyration and area-weighted moments provide the firmest evidence to date for the actual existence of a leading non-analytic correction to scaling exponent $\Delta=3 / 2$, thus confirming the theoretical predictions made by Nienhuis [14].

\section{Conclusion}

We have presented an improved algorithm for the calculation of the radius of gyration and area-weighted moments of self-avoiding polygons on the square lattice. This algorithm has enabled us to calculate these series for polygons up to perimeter length 82. Our extended series enables us to give very precise estimate of the critical exponents, which are consistent with the exact values $\alpha=1 / 2$ and $\nu=3 / 4$. We also obtain a very precise estimate for the amplitude $B=0.5623012(1)$. Analysis of the coefficients of the radius of gyration series yielded results fully compatible with the prediction $B D=5 / 16 \pi^{2}$. This allows us to obtain the very accurate estimate $D=0.05630944(1)$. From the first areaweighted moment we obtained the estimate $E^{(1)}=0.141520(2)$, which allows us to give a much improved estimate for the universal amplitude ratio $E^{(1)} / D=2.51326(3)$. We also find firm evidence for the existence of a non-analytic correction-to-scaling term with exponent $\Delta=3 / 2$.

\section{E-mail or WWW retrieval of series}

The series for the various generating functions studied in this paper can be obtained via e-mail by sending a request to I.Jensen@ms.unimelb.edu.au or via the world wide web on the URL http://www.ms.unimelb.edu.au/ iwan/ by following the instructions. 


\section{Acknowledgements}

Thanks to Tony Guttmann for many valuable comments on the manuscript and the series analysis. Financial support from the Australian Research Council is gratefully acknowledged.

\section{References}

[1] B. D. Hughes, in Random walks and random environments, Vol. I Random walks, Clarendon Press, Oxford (1995).

[2] I. G. Enting, J. Phys. A. 13, 3713 (1980).

[3] I. G. Enting and A. J. Guttmann, J. Phys. A. 18, 1007 (1985).

[4] A. J. Guttmann and I. G. Enting, J. Phys. A. 21, L165 (1988).

[5] B. J. Hiley and M. F. Sykes, J. Chem. Phys. 34, 1531 (1961).

[6] I. G. Enting and A. J. Guttmann, J. Stat. Phys. 58, 475 (1990).

[7] V. Privman and J. Rudnick, J. Phys. A. 18, L789 (1985).

[8] I. Jensen and A. J. Guttmann, J. Phys. A. 32, 4867 (1999).

[9] A. R. Conway, J. Phys. A. 28, 335 (1995).

[10] P. G. de Gennes, Phys. Lett. 38A, 339 (1972).

[11] J. des Cloizeaux, J. Physique 36, 281 (1975).

[12] F. Family, Phys. Lett. 92A, 341 (1982).

[13] S. Leibler, R. R. P. Singh and M. E. Fisher, Phys. Rev. Lett. 59, 1989 (1987).

[14] B. Nienhuis, Phys. Rev. Lett. 49, 1062 (1982).

[15] J. L. Cardy and A. J. Guttmann, J. Phys. A. 26, 2485 (1993).

[16] K. Y. Lin and S.-J. Lue, Physica A 270, 453 (1999).

[17] A. J. Guttmann, in Phase Transitions and Critical Phenomena, Vol. 13, eds. C Domb and J L Lebowitz, Academic Press, New York (1989).

[18] A. R. Conway and A. J. Guttmann, Phys. Rev. Lett. 77, 5284 (1996). 
Table 1: The mean-square radius of gyration, first and second area-moments of $n$-step self-avoiding polygons on the square lattice. Only non-zero terms are listed.

\begin{tabular}{|c|c|c|c|}
\hline$n$ & $p_{n} n^{2}\left\langle R^{2}\right\rangle_{n}$ & $p_{n}\langle a\rangle_{n}$ & $p_{n}\left\langle a^{2}\right\rangle_{n}$ \\
\hline 4 & 8 & 1 & 1 \\
\hline 6 & 66 & 4 & 8 \\
\hline 8 & 600 & 22 & 70 \\
\hline 10 & 5164 & 124 & 560 \\
\hline 12 & 42872 & 726 & 4358 \\
\hline 14 & 346828 & 4352 & 33160 \\
\hline 16 & 2754056 & 26614 & 248998 \\
\hline 18 & 21549780 & 165204 & 1851040 \\
\hline 20 & 166626744 & 1037672 & 13655432 \\
\hline 22 & 1275865332 & 6580424 & 100126648 \\
\hline 24 & 9690096824 & 42062040 & 730548788 \\
\hline 26 & 73090383120 & 270661328 & 5308524968 \\
\hline 28 & 548064459968 & 1751614248 & 38442000664 \\
\hline 30 & 4088719617824 & 11391756176 & 277565593032 \\
\hline 32 & 30367415294800 & 74406502814 & 1999068564026 \\
\hline 34 & 224659143155964 & 487838450116 & 14365917755936 \\
\hline 36 & 1656259765448200 & 3209229661682 & 103038218758426 \\
\hline 38 & 12172580326973688 & 21175301453040 & 737765745264544 \\
\hline 40 & 89212147340159520 & 140097533633112 & 5274413814993896 \\
\hline 42 & 652183776123444404 & 929160187609096 & 37655943519835560 \\
\hline 44 & 4756877451862073312 & 6176075676719784 & 268506373782824280 \\
\hline 46 & 34623252929242595840 & 41135052992574928 & 1912438211281990104 \\
\hline 48 & 251526960780642980968 & 274482801972069490 & 13607405560541031042 \\
\hline 50 & 1824061566724351292496 & 1834665820375683428 & 96728883661202188552 \\
\hline 52 & 13206639904144205117592 & 12282315178525359966 & 687010148492686667614 \\
\hline 54 & 95476389002729304216548 & 82344395405972692656 & 4875571799890192459056 \\
\hline 56 & 689283065294740945143208 & 552806313387704627982 & 34575571741149137524846 \\
\hline 58 & 4969805963839723557919424 & 3715834986939390916244 & 245029144855912573003776 \\
\hline 60 & 35789811145967164348552960 & 25006203000374020526746 & 1735367234605432029439794 \\
\hline 62 & 257449325423816274956954508 & 168466668960946012707912 & 12283126555855361655011856 \\
\hline 64 & 1849981836861769186990365288 & 1136122707072612282498874 & 86893466632100569644163186 \\
\hline 66 & 13280506839637150191613774736 & 7669275741518968346891172 & 614385797629196735502076968 \\
\hline 68 & 95248670945282200958664147712 & 51817515409677258092083006 & 4341950222145487318409546446 \\
\hline 70 & 682533032784692897614712920788 & 350404221555935013278573224 & 30671194434233707728683946784 \\
\hline 72 & 4886864684580008620898035643960 & 2371438542131929578320200646 & 216565948566766116053230547838 \\
\hline 74 & 34962179240623880562564354461036 & 16061466455829089444235194204 & 1528529336761773075102657075616 \\
\hline 76 & 249946063483045736235271147799248 & 108860864860439323866007261128 & 10784279532727353410458586600848 \\
\hline 78 & 1785625611982607482936563853493112 & 738338427155234332385671368928 & 76059086282576056911156299311952 \\
\hline 80 & 12748122227351375676612377672210416 & 5010964557143508508512736679936 & 536243262589039476652829061618528 \\
\hline 82 & 90955298658999234326739061737970500 & 34029495976431151261075225822320 & 3779470144925357385934811283997288 \\
\hline
\end{tabular}

Table 2: Estimates for the critical point $u_{c}$ and exponents obtained from second order differential approximants to the series for the radius of gyration, first and second moments of area of square lattice self-avoiding polygons. $L$ is the order of the inhomogeneous polynomial.

\begin{tabular}{|c|c|c|c|c|c|c|}
\hline \multirow{2}{*}{$\begin{array}{l}\text { Series: } \\
L\end{array}$} & \multicolumn{2}{|c|}{$\overline{\mathcal{R}_{g}^{2}(u)}$} & \multicolumn{2}{|c|}{$\overline{\mathcal{P}^{(1)}(u)}$} & \multicolumn{2}{|c|}{$\overline{\mathcal{P}^{(2)}(u)}$} \\
\hline & $u_{c}$ & $-(\alpha+2 \nu)$ & $u_{c}$ & $2-(\alpha+2 \nu)$ & $u_{c}$ & $2-(\alpha+4 \nu)$ \\
\hline 0 & $0.14368045(10)$ & $-1.99941(23)$ & $0.143680543(86)$ & $0.00027(19)$ & $0.143680502(35)$ & $-1.499523(78)$ \\
\hline 1 & $0.14368057(14)$ & $-1.99976(54)$ & $0.143680556(33)$ & $0.000279(78)$ & $0.143680539(19)$ & $-1.499662(62)$ \\
\hline 2 & $0.14368063(13)$ & $-1.99997(58)$ & $0.143680558(31)$ & $0.000266(66)$ & $0.143680535(22)$ & $-1.499658(86)$ \\
\hline 3 & $0.14368048(11)$ & $-1.99948(21)$ & $0.143680562(25)$ & $0.000267(48)$ & $0.143680530(20)$ & $-1.49958(21)$ \\
\hline 4 & $0.143680540(71)$ & $-1.99956(18)$ & $0.143680567(25)$ & $0.000253(57)$ & $0.143680541(21)$ & $-1.499636(57)$ \\
\hline 5 & $0.143680553(60)$ & $-1.99959(18)$ & $0.143680566(27)$ & $0.000254(66)$ & $0.143680545(15)$ & $-1.499654(28)$ \\
\hline 6 & $0.143680542(33)$ & $-1.999544(92)$ & $0.143680577(13)$ & $0.00018(17)$ & $0.143680542(15)$ & $-1.499649(58)$ \\
\hline 7 & $0.14368046(10)$ & $-1.99942(14)$ & $0.143680564(23)$ & $0.000255(58)$ & $0.143680542(16)$ & $-1.499658(64)$ \\
\hline 8 & $0.143680511(43)$ & $-1.999474(96)$ & $0.143680568(21)$ & $0.000246(48)$ & $0.143680539(17)$ & $-1.499650(57)$ \\
\hline 9 & $0.143680527(64)$ & $-1.99952(15)$ & $0.1436805828(92)$ & $0.000214(30)$ & $0.143680548(28)$ & $-1.499674(71)$ \\
\hline 10 & $0.143680511(41)$ & $-1.999472(94)$ & $0.143680572(17)^{\prime}$ & $0.000238(43)$ & $0.143680544(16)$ & $-1.499665(52)$ \\
\hline
\end{tabular}



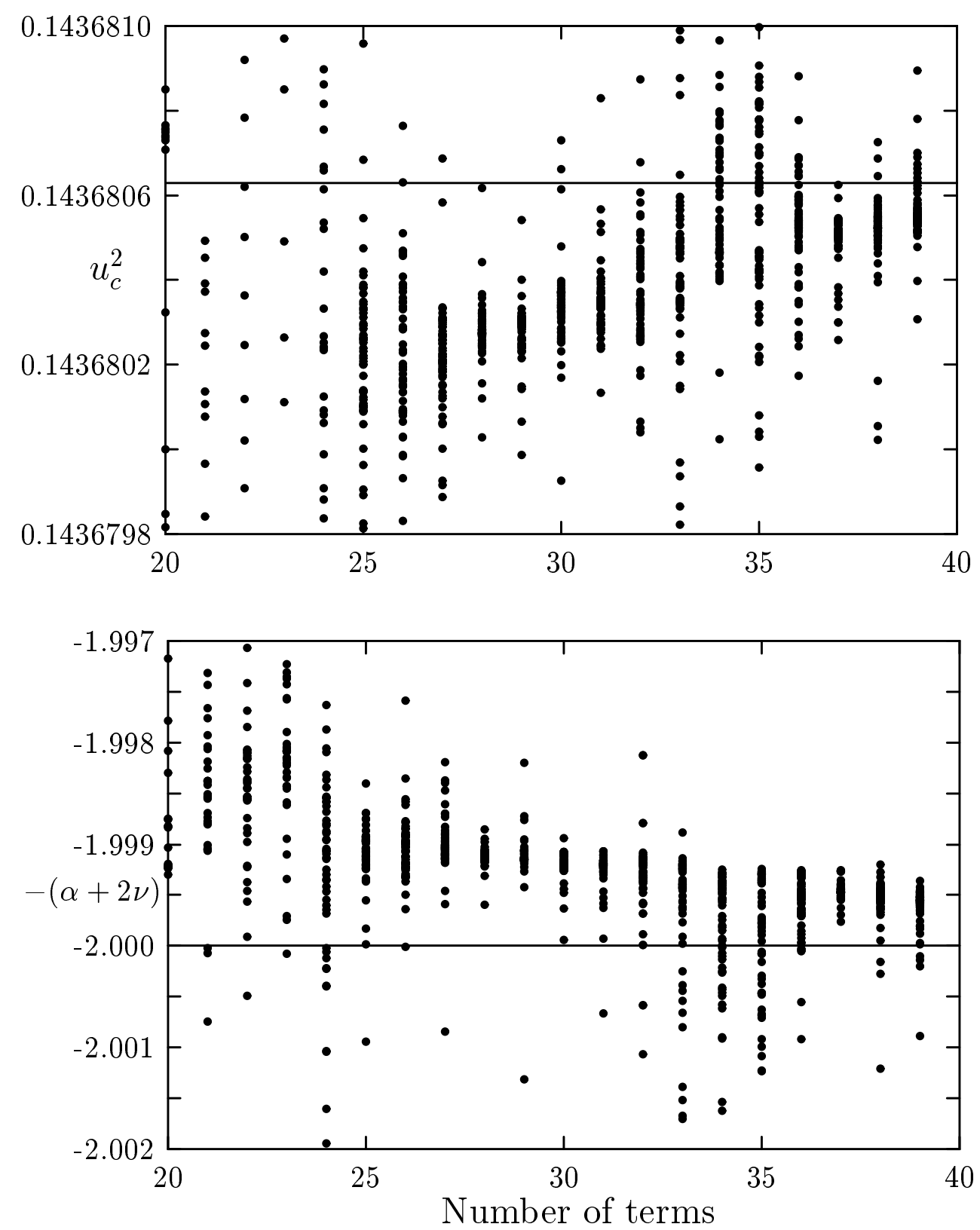

Figure 1: Estimates for the critical point and exponent of the generating function for the radius of gyration of square lattice polygons as a function of the number of terms used by the second order differential approximants. The solid lines indicate the expected values $u_{c}=0.14368062927(1)$ and $\xi=-(\alpha+2 \nu)=-2$. 

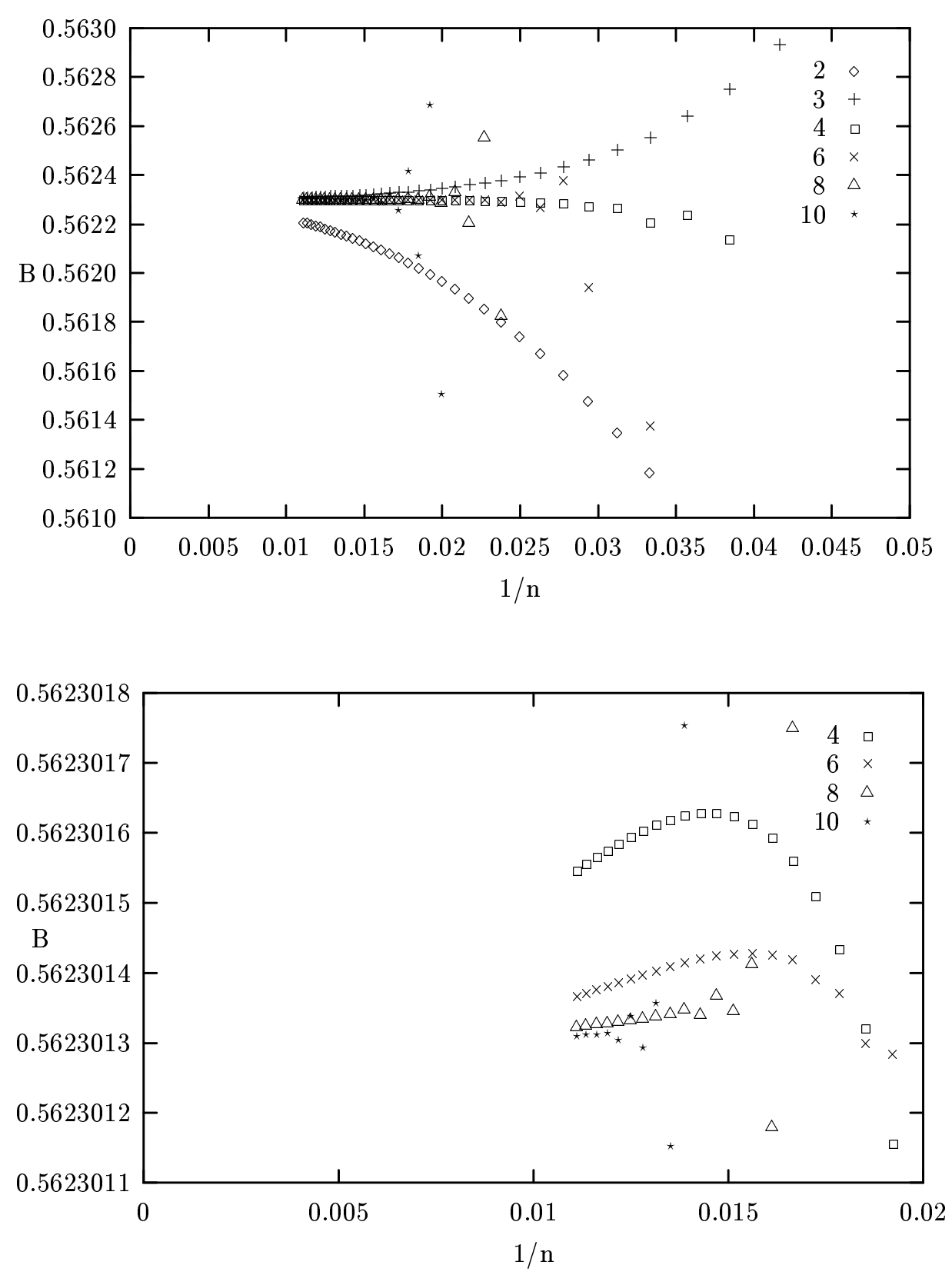

Figure 2: Estimates for the leading amplitude $a_{0}=B$ of square lattice polygons as a function of $1 / n$. Each data set is obtained by fitting $p_{n}$ to the form given in equation (11) using from 2 to 10 correction terms. The lower panel displays a detailed look at the data in the upper panel. 

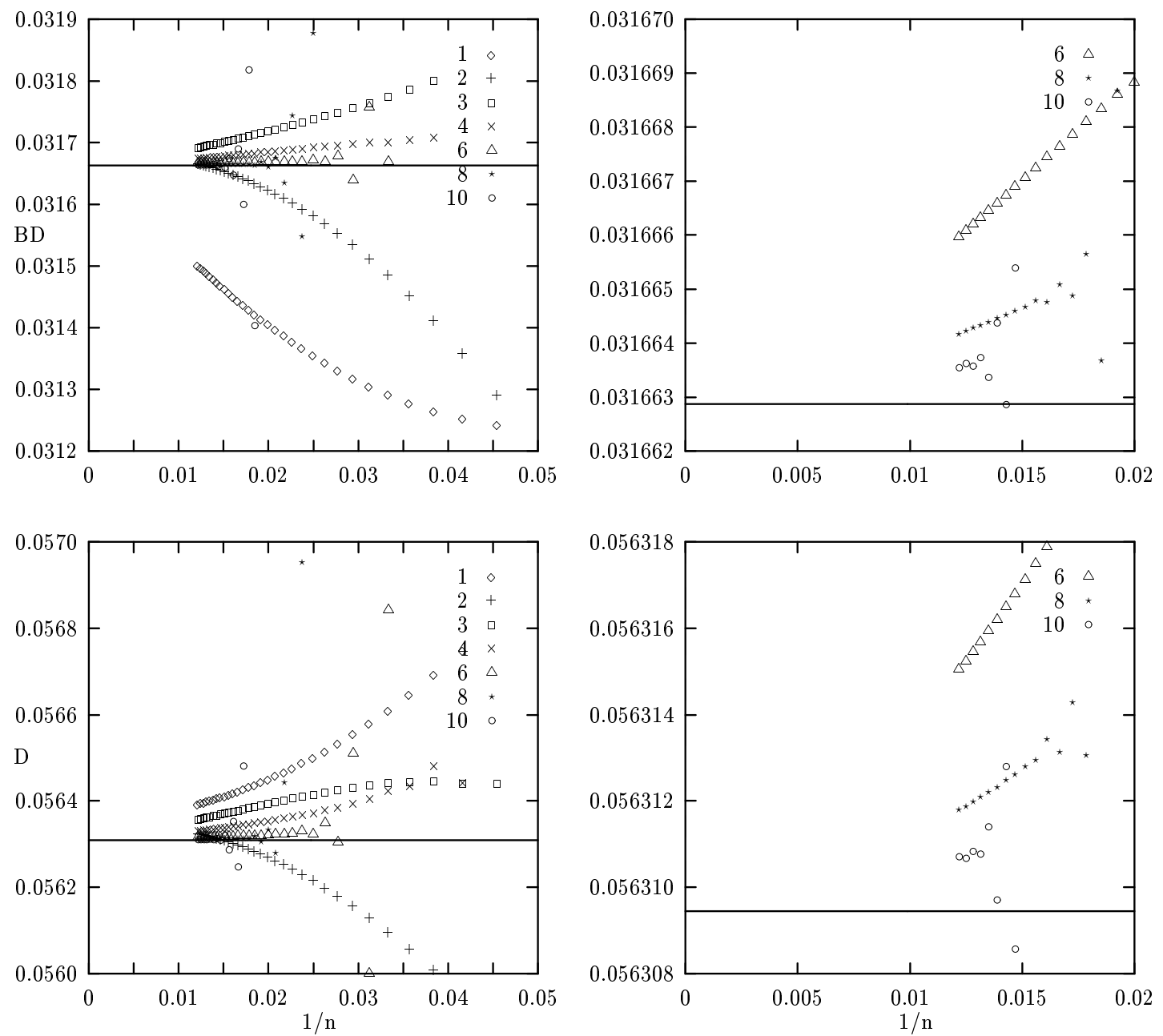

Figure 3: Estimates for the leading amplitude $B D$ and $D$ of the radius of gyration of square lattice polygons as a function of $1 / n$. Each data set in the top panels is obtained by fitting the coefficients $r_{n}$ of the radius of gyration generating function to the form given in equation (12), using from 1 to 10 correction terms. Each data set in the bottom panels is obtained by fitting $r_{n} / p_{n}$ to the form given in equation (13). The right panels are a detailed look at the data in the left panels. The solid lines indicate the expected values given in the text. 